\title{
Design Study of the GNIRS Bracket Structure
}

\author{
Myung Cho \\ Optical Sciences Center, University of Arizona and Gemini $8 m$ Telescopes \\ Project, Tucson AZ 85726 \\ Chun C. Li, Woon-Yin Wong, Randy Cuberly \\ National Optical Astronomy Observatories, 950 N Cherry, Tucson, AZ 85726 \\ Il K. Moon \\ Civil Engineering and Engineering Mechanics, University of Arizona, Tucson, \\ AZ 85719
}

Gemini Preprint \# 29 


\title{
Design Study of the GNIRS Bracket Structure
}

\author{
Myung K. Cho ${ }^{\mathrm{a}}$, Chun C. Li ${ }^{\mathrm{b}}$, Woon-Yin Wong ${ }^{\mathrm{b}}$, Randy Cuberly ${ }^{\mathrm{b}}$, Il K. Moon ${ }^{\mathrm{c}}$ \\ ${ }^{a}$ Optical Sciences Center, University of Arizona and Gemini 8m Telescopes Project, Tucson AZ 85726 \\ ${ }^{\mathrm{b}}$ National Optical Astronomy Observatories, 950 N Cherry, Tucson, AZ 85726 \\ ${ }^{\mathrm{c}}$ Civil Engineering and Engineering Mechanics, University of Arizona
}

\begin{abstract}
The internal support structure of the Gemini Near Infrared Spectrograph (GNIRS) comprises a series of substructures (modules) which are interconnected to support the optical components and their mechanisms. A very stable support structure is required in the GNIRS to exploit the high image quality of the Gemini telescope. The initial concept for the structure used triangular trusses connecting stiff circular rings; this type of structure did not provide sufficient stiffness. This concept was replaced by a novel type of structure employing lightweight cylindrical modules, with each module produced by numerically controlled machining from a solid. Finite element analysis is combined with three-dimensional layout techniques to develop an optimized structural configuration for each module. A parametric process was performed for the design optimization to produce the highest fundamental frequency for a given weight, as well as to deal with the normal concerns about global deformation and stress.
\end{abstract}

Keywords: large telescope, instrument bracket structure, Spectrograph, lightweight, deformation and stress

\section{INTRODUCTION}

Support structures for large optical instruments have always challenged scientists and engineers in design and fabrication ${ }^{1}$. Because they are massive and complicated, the design has often been overly conservative. As a classical design, Serrurier truss structures are widely adopted because of simplicity, and are based on a concept of balancing masses ${ }^{2}$. This concept may be applicable to relatively simple optical configurations, or where the mass and size of the instrument are not driving design considerations. Serrurier truss support works adequately with systems of uniform mass distribution or of symmetric configuration.

The GNIRS is designed to provide high-precision optical performance to match the state-of-art Gemini 8-m Telescope's superb image quality. It is a quite complicated and massive optical instrument, with about 30 optical components, and its optical package weighs about 8,000 Newton. All optical components are cooled to below $70 \mathrm{~K}$, so the support structure must provide thermal isolation without sacrificing rigidity. An extremely stable internal support structure is required in the GNIRS to exploit the high image quality of the Gemini telescope. The initial design concept for the support structure utilized truss members. The trusses were connected to a stiff circular ring in the center, as in a Serrurier truss. It was found that this type of structure did not provide sufficient stiffness. This concept was replaced by a novel type of structure employing lightweight cylindrical modules, with each module produced by numerically controlled machining from a solid. The support structure comprises a series of interconnected substructures which support the optical components and their mechanisms. This modular substructure provides excellent serviceability during handling, assembling, and disassembling procedures. It provides great ease in the manufacturing process and avoids potential risks during many operations.

Finite element analysis is mainly utilized to develop an optimized structural configuration for each module. A parametric process was performed for the design optimization to produce the highest fundamental frequency for a given weight, in addition to the normal concerns about global deformation and stress. The optical layout of the GNIRS with a short-focallength camera is shown in Figure 1. 


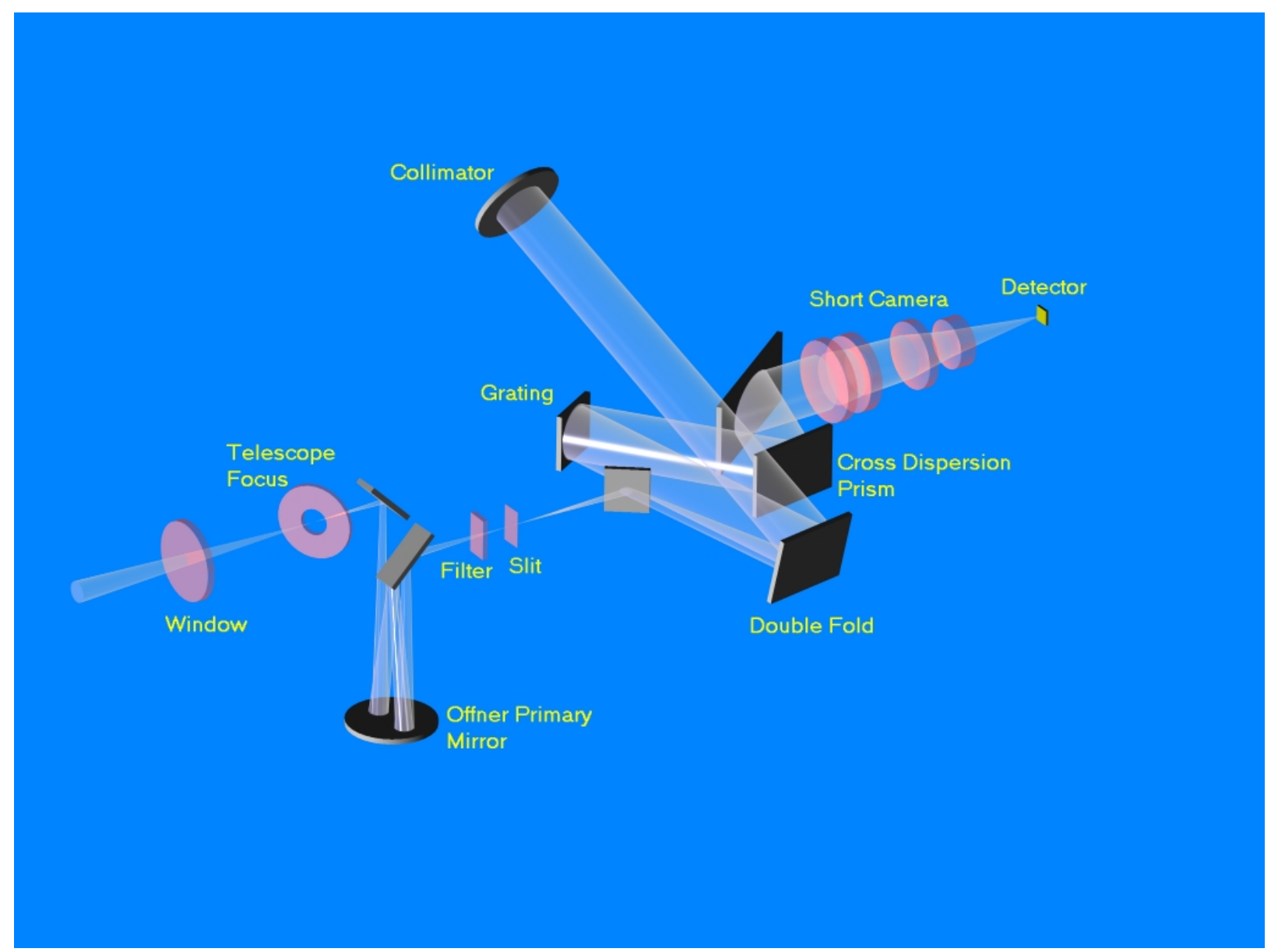

Figure 1. Optical Layout of Gemini Near-Infrared Spectrograph with a Short-focal-length Camera

\section{BASELINE MODULE}

The support structure of the GNIRS comprises a thermal isolation cylinder, a total of five cylindrical modules, and a camera module. The main part of the support system is the five cylindrical modules made out of aluminum (AL 6061 T651). Each of these modules is designed to include the optical systems and their mounting mechanisms based on their positions and orientations. A baseline module was carefully studied to account for the structural functions of the individual modules. Each module is further modified and adjusted from the baseline module taking its individual functions. The baseline module was required to have high stiffness relative to its weight. The module was optimized based on the following design parameters (see Figure 2):

\section{Fundamental frequency (mass and structural stiffness)}

Wall thickness of the cylinder, wall thickness of the grid cell (pocket), and plate thickness of the mid-plane

Size of the pocket and shape of the pocket

Number of primary connectors (studs) and number of secondary connectors (bolts)

Thermal considerations and the contact area (structurally and thermally)

Machineability

Table 1 lists the mass and the fundamental frequency of a baseline module at a typical range of parameters for a uniform cell grid size of $100 \mathrm{~mm}$ by $100 \mathrm{~mm}$. Three different configurations in the wall thickness of the cylinder $(6 \mathrm{~mm}, 9 \mathrm{~mm}$, and $12 \mathrm{~mm}$ ) were examined, as well as various wall thicknesses of the grid pattern and the mid plates. 
Table 1. Mass and fundamental frequency of a baseline module for various wall thickness

Cylinder wall thickness $=6 \mathrm{~mm}$

\begin{tabular}{|l|c|c|c|}
\hline Grid wall thickness & $6 \mathrm{~mm}$ & $9 \mathrm{~mm}$ & $12 \mathrm{~mm}$ \\
\hline Mass $(\mathrm{kg})$ & 95 & 136 & 176 \\
\hline Frequency (hz) & 423 & 408 & 397 \\
\hline
\end{tabular}

Cylinder wall thickness $=9 \mathrm{~mm}$

\begin{tabular}{|l|c|c|c|}
\hline Grid wall thickness & $6 \mathrm{~mm}$ & $9 \mathrm{~mm}$ & $12 \mathrm{~mm}$ \\
\hline Mass $(\mathrm{kg})$ & 101 & 142 & 183 \\
\hline Frequency (hz) & 441 & 425 & 414 \\
\hline
\end{tabular}

Cylinder wall thickness $=12 \mathrm{~mm}$

\begin{tabular}{|l|c|c|c|}
\hline Grid wall thickness & $6 \mathrm{~mm}$ & $9 \mathrm{~mm}$ & $12 \mathrm{~mm}$ \\
\hline Mass $(\mathrm{kg})$ & 107 & 148 & 190 \\
\hline Frequency (hz) & 454 & 437 & 427 \\
\hline
\end{tabular}

Parametric design iterations determined an optimal configuration for the baseline module. This basic configuration is: overall diameter $=1100 \mathrm{~mm}$, overall height $=224 \mathrm{~mm}$, cylinder wall thickness $=12 \mathrm{~mm}$, pocket wall thickness $=6 \mathrm{~mm}$, mid plate thickness $=6 \mathrm{~mm}$, and light weight pocket shape configuration $=150 \mathrm{~mm}$ by $150 \mathrm{~mm}$ square. The module height was set by the fabrication procedures for the module. With a common NC machine, an aluminum alloy disk of $1100 \mathrm{~mm}$ in diameter and $250 \mathrm{~mm}$ deep can be handled adequately.

Proper preloading of threaded fasteners is critical to their performance in the assembly. The preload necessary for the fasteners is mainly governed by the structural stiffness and the temperature variation in the assembly. A set of five (5) primary studs is used for structural joint between the modules. These studs are custom made ones similar to M18 or equivalent bolts, and are designed to carry most of the structural loads. Additionally, there is a set of 60 secondary bolts (12 M6 bolts between the primary joints) on the interface flange. The main purpose of these bolts is to provide a better thermal interface between modules. A pair of shear pins will be installed in each module. These pins will carry the shear forces induced by the weight of modules and direct or indirect sources during handling and operation. The diameter of the pins is $18 \mathrm{~mm}$. A sketch of the baseline module is shown in Figure 2.

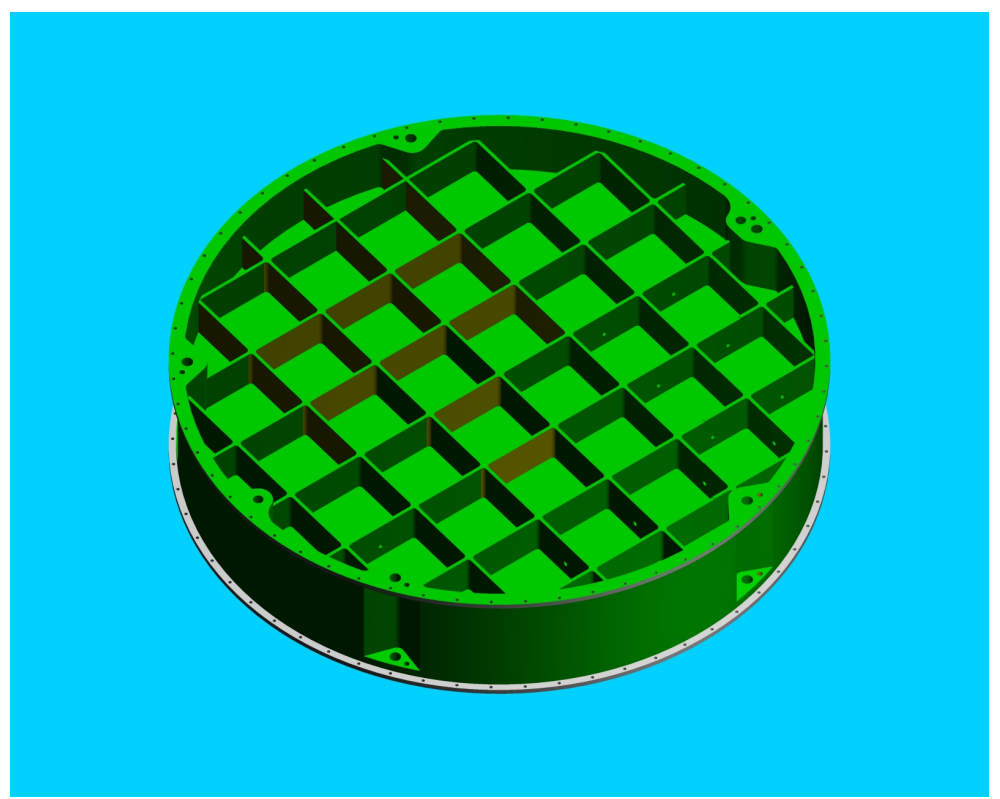

Figure 2. Baseline Module 


\section{STRUCTURAL ANALYSIS}

The GNIRS was designed to meet tight scientific functional constraints. During a one hour exposure, the maximum permissible motion of the slit image at the detector is 0.1 pixel, or about 2.7 microns. This demanding specification on the flexure of the instrument corresponds to the effect of a 15 degree rotation of the gravity vector during the period of observation. A very stiff structure is required to meet this specification. The GNIRS fundamental frequency goal is set for $100 \mathrm{hz}$, which corresponds to a global deflection of about 30 microns at the instrument as it is moved from the zenith to horizon.

\subsection{Modules and Subassemblies}

The support structure of GNIRS is designed to support all the optical components and the subassemblies. The GNIRS has a typical internal operational temperature of $65 \mathrm{~K}$. It is also under vacuum with up to one atmospheric pressure difference between the inside and the outside of the dewar. In order to reduce thermal radiation effects in the mechanism, there are two passive shields and one actively cooled shield around the support structure. To minimize the thermal convection into the support structure, there is a thermal insulation cylinder - a cylinder made out of a filament-wound G10 fiberglass. This cylinder has two functions. First, it provides structural stiffness as a main structural member which is attached to the interface support structure (ISS) plate at one end and to the interface location between Module 1 and Module 2 at the other. Secondly, it provides thermal isolation inside the support structure. A trade off study was performed to determine the length and wall thickness based on structural performance and thermal characteristics. Final configurations of the G10 cylinder are: $1100 \mathrm{~mm}$ diameter, $524 \mathrm{~mm}$ length, and $18 \mathrm{~mm}$ wall thickness. The estimated mass of this cylinder is $60 \mathrm{~kg}$. Typical mechanical and thermal properties of the G10 used in the study are as follows:

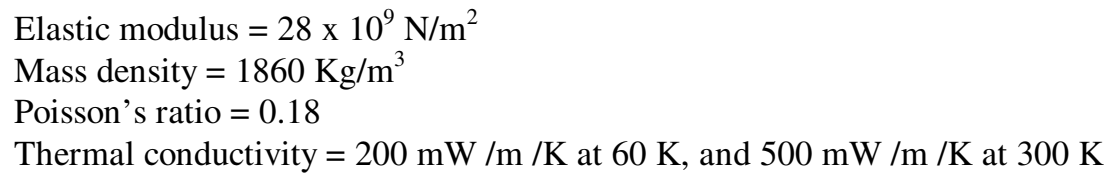

The first module behind the G10 cylinder is called Module 1. It is designed to mount an Offner relay assembly and an OIWFS (on instrument wavefront sensor). The Offner assembly is a two-mirror relay system which includes a cold stop where the telescope secondary is re-imaged. The support system of the Offner primary mirror uses a flexure mount to reduce effects from the thermal mismatch between the aluminum mirror substrate and the optical reflective coating material (electroless nickel or aluminum plate). This effect is known as "bimetallic bending". The OIWFS package comprises several subassemblies. It provides tip-tilt correction and fine focus correction to facilitate acquisition of objects. The OIWFS consists of a pickup mirror unit and a gimbal mirror assembly. The entire package is to be mounted either directly onto Module 1 or on an extended secondary support structure (Module 0 - provisional). Module 1 provides sufficient stiffness for the mounts and accurate positioning precision for the optical systems. Additionally, this module includes a baffle mechanism to control stray light. The estimated mass of this module itself is $59 \mathrm{~kg}$, and the mass of the Offner including the optics and the mounts is $28 \mathrm{~kg}$. The OIWFS package was a mass of approximately $100 \mathrm{~kg}$. Module 1 and the Offner assembly are shown in Figure 3. The finite element model of the module is also shown in the figure. In the finite element analysis, a lumped mass was used for this module assembly.

On Module 2, a pair of filter wheels and slit and decker wheels are mounted. The pair of filter wheels contain 22 filters for order sorting and acquisition. The estimated mass of the filter wheels is $32 \mathrm{~kg}$, which includes the mechanism mount and the motor drive units. Discrete slits, etched into metal plates, are placed in a slit wheel. The slit length is varied by a second set of fixed apertures placed behind the slit. Since the light relayed from the Offner re-images the focus of the telescope onto the entrance slit, the precise position of the slit is extremely important. There is an access hole in the outer wall of the module for servicing the filters and the slits. The estimated mass of the slit wheel is $24 \mathrm{~kg}$, which includes the mechanism mount and the motor drive units. The module, filter, and slit wheel assemblies are shown in Figure 4. The finite element model of the module is also shown in the same figure. For the finite element analysis, lumped masses were used. 


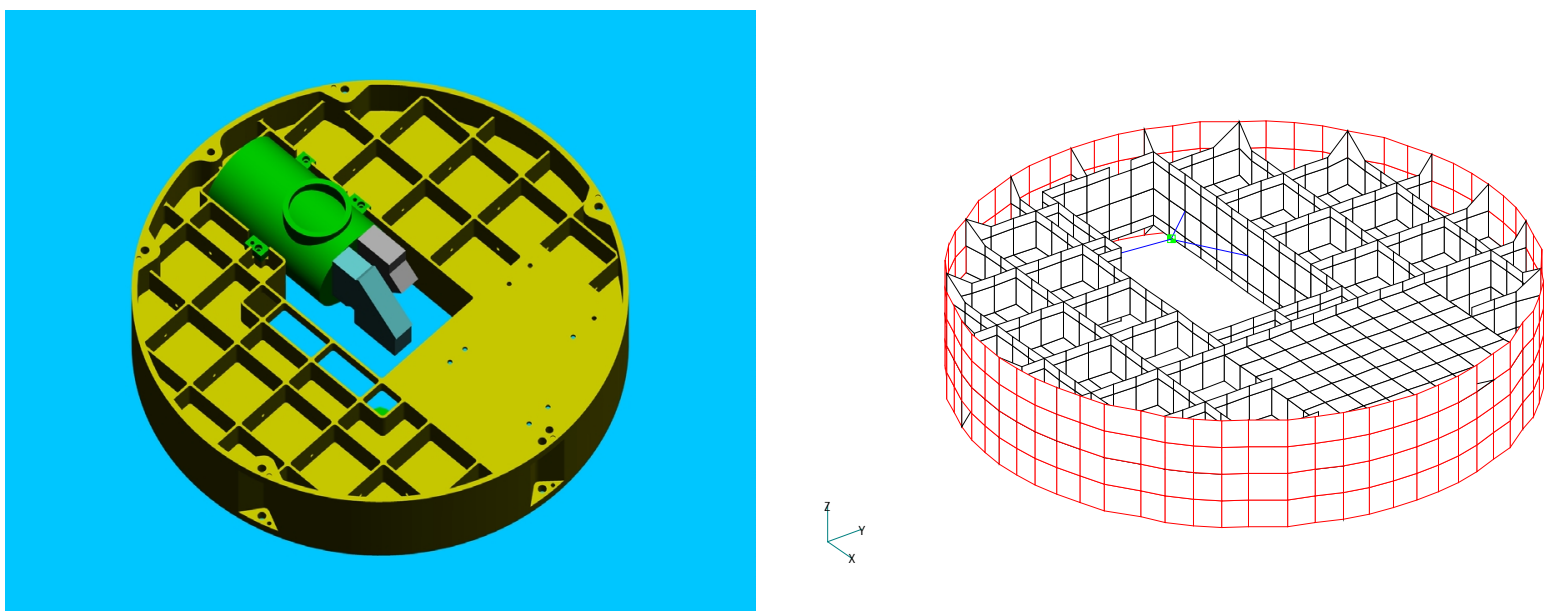

Figure 3. Module 1
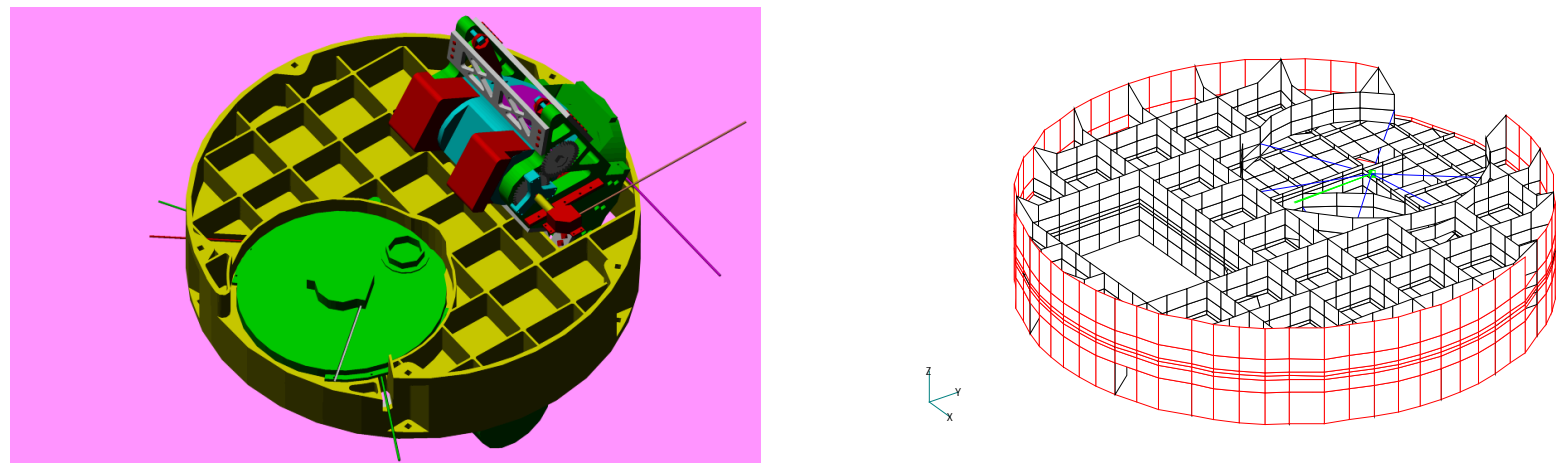

Figure 4. Module 2

Module 3 is the most complicated one. The grating turret, two fold mirrors (a small fold mirror and a large double fold mirror), and the collimator are mounted on this module. The collimator mirror is an off-axis parabola and is held by a kinematic three point support system. This support mechanism is also designed to have a five-degree-of-freedom adjustment for initial alignment. The estimated mass of the collimator assembly is about $7 \mathrm{~kg}$. This assembly is rather large, so it must be mounted on both Module 3 and Module 4. The grating turret assembly provides selection of the grating and tilt of the selected grating, with two independent axes. It contains four gratings and one flat mirror. The grating turret is located on the opposite side of the collimator on the module. An extremely stiff mounting support for this mechanism is required because of the size and the mass. Additionally, the turret requires baffling for stray light and accommodates a tilt motion of seven (7) degrees from the axis. The total mass of the grating turret was estimated as 80 $\mathrm{kg}$. The double fold mirror is a flat with semi-octagonal shape. It is held by a flexure mount and its estimated mass is 8 $\mathrm{kg}$. This is about as large as the collimator, and like the collimator is partially mounted onto Module 4. The module, mirrors, and grating assemblies are shown in Figure 5. The finite element model of the module is shown as well.

Both the collimator mirror and the double fold mirror assemblies are partially mounted on Module 4. A cross-dispersion turret is also installed on this module. The cross-dispersion turret has two cross-dispersion prisms. The estimated mass of this cross dispersion unit is $50 \mathrm{~kg}$. The collimator mirror and the double fold mirrors are to be installed from outside of the module. This module was designed to account for complicated light paths of incoming light and outgoing light between the optics - the double fold mirror, the collimator, the grating, the cross dispersion prism. Significant amounts of effort in design and analysis were made for the stray light baffling. The finite element model of this module and the design illustration are shown in Figure 6. 
Module 5 includes the fold mirror 7, a large flat mirror, and a sizable cavity for the cross dispersion turret. The fold mirror relays the light from the cross dispersion turret to the camera turret assembly. The mass of the folding mirror is estimated as $6 \mathrm{~kg}$. The module layout and the finite element model are shown in Figure 7.

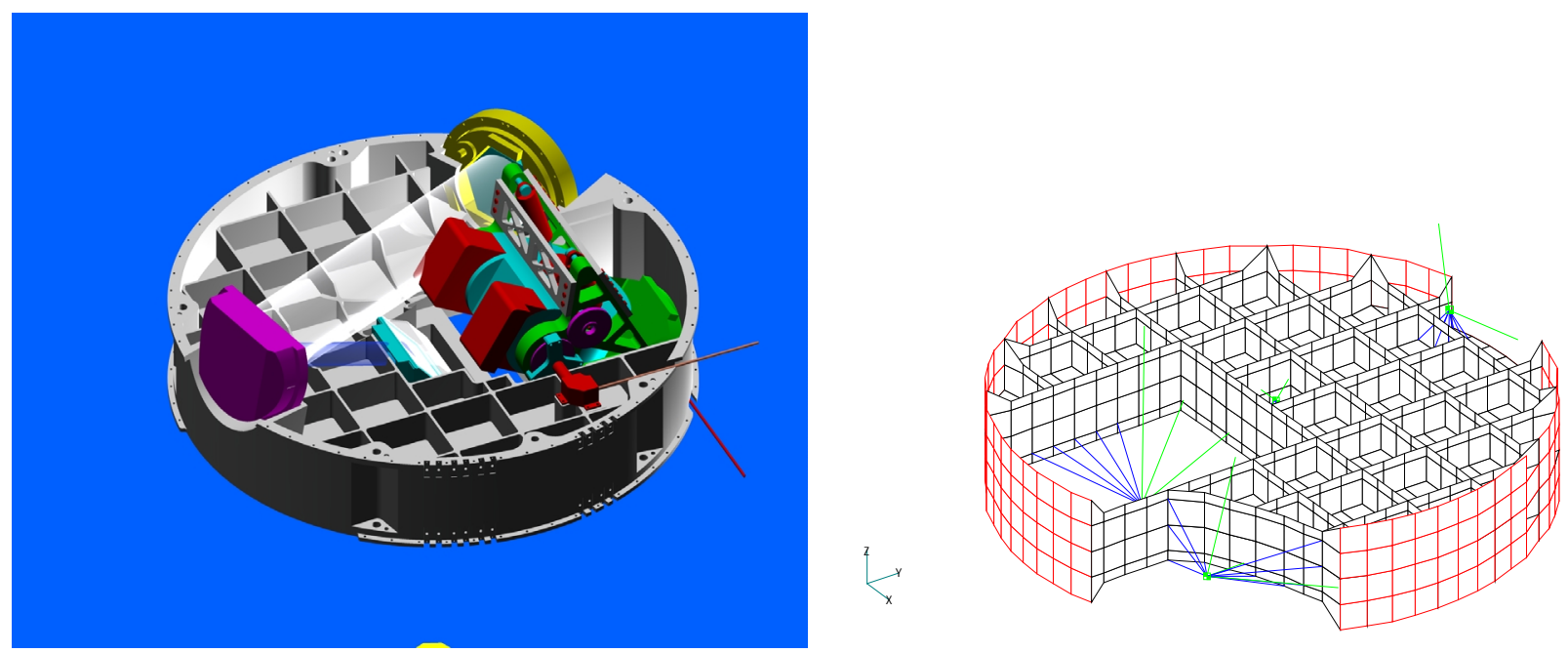

Figure 5. Module 3
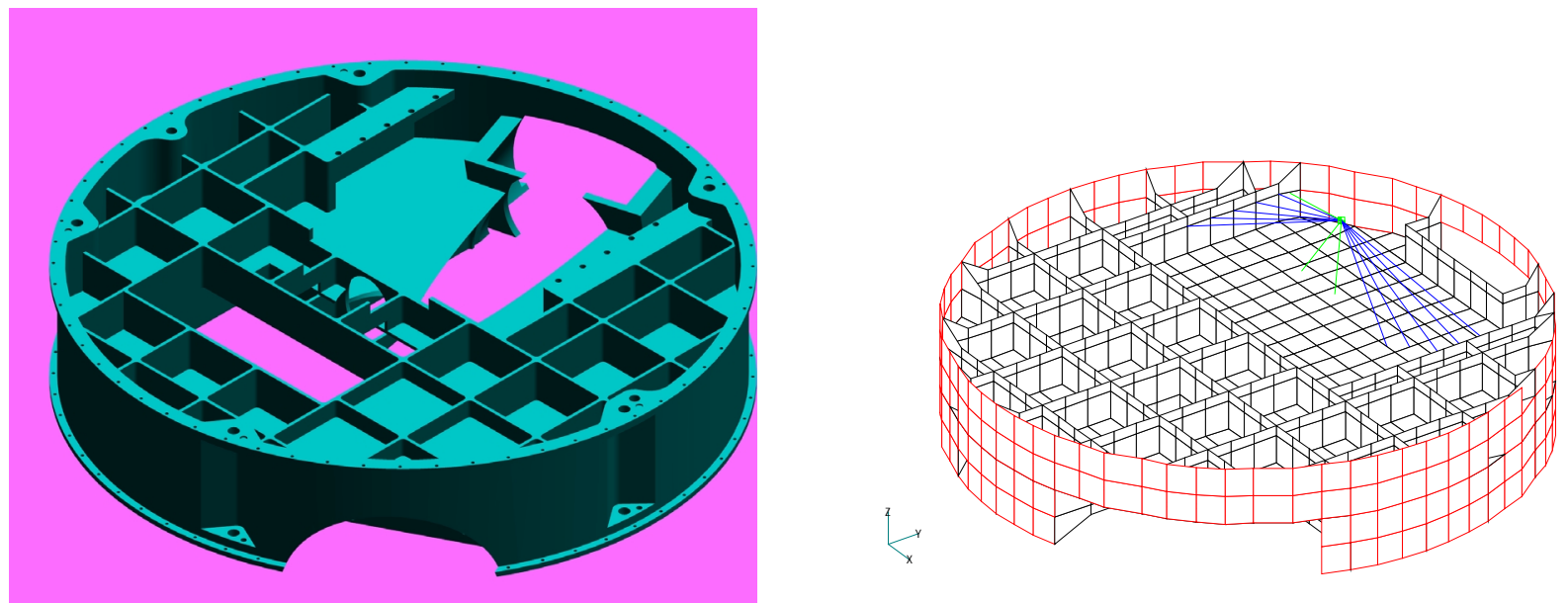

Figure 6. Module 4
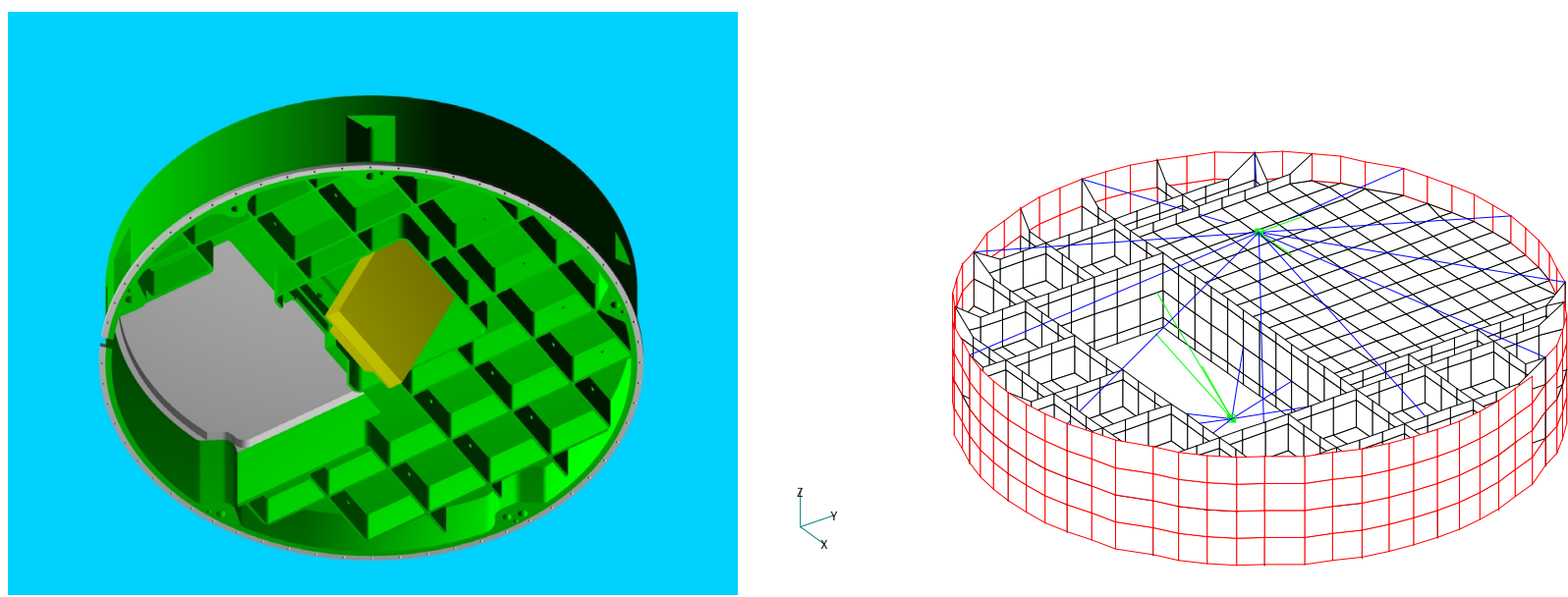

Figure 7. Module 5 
The camera module provides a support system for a camera assembly. It is a relatively long cylindrical housing and is about $60 \%$ of the diameter of the other modules. The camera turret assembly is mounted on an interface plate located on the top surface of Module 5. There are four cameras mounted inside the camera turret. The estimated mass of the camera assembly is $85 \mathrm{~kg}$. The module layout and the finite element model are shown in Figure 8.

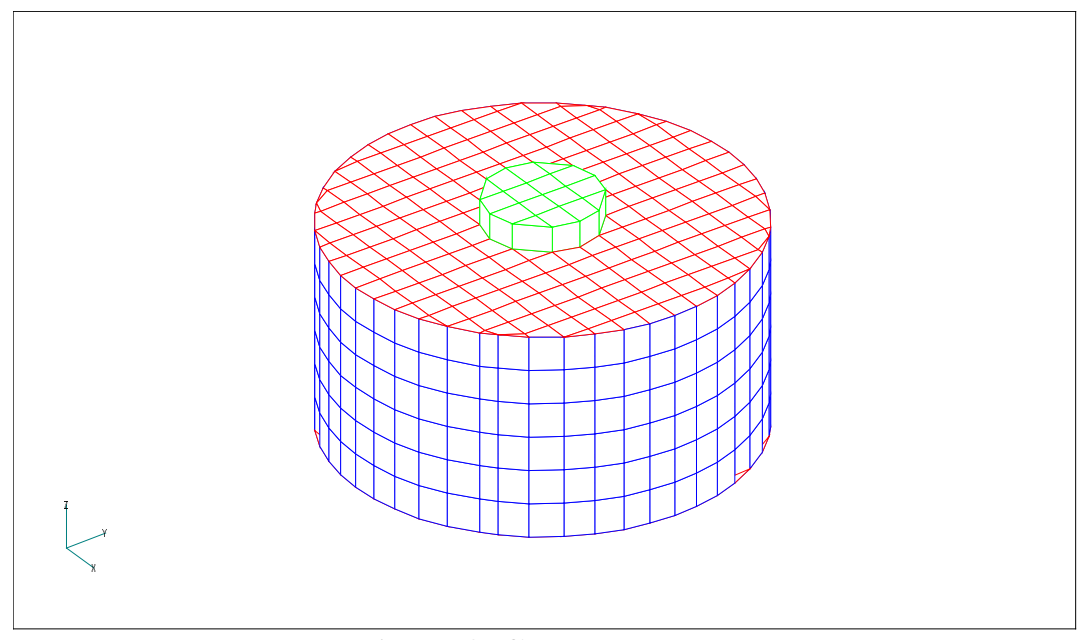

Figure 8. Camera Module

For each module, the mass of the optical components including their mounting mechanisms and their nominal dimensions are listed in Table 2.

Table 2: Mass and dimensions of the individual module

\begin{tabular}{|l|c|c|}
\hline & Mass (Kg) & Dimension (mm) \\
\hline G10 & 60.4 & D550 x L525.262 x T18 \\
\hline
\end{tabular}

\begin{tabular}{|l|c|c|}
\hline Module 1 & 58.5 & D1100 x T224 \\
\hline Offner & 28 & D199.2 x T18.75 \\
\hline
\end{tabular}

\begin{tabular}{|l|c|c|}
\hline Module 2 & 61.2 & D1100 x T224 \\
\hline Slit Wheel & 23.6 & D500 x T25 \\
\hline Filter Wheel & 32 & D528.125 x T40 \\
\hline
\end{tabular}

\begin{tabular}{|l|c|c|}
\hline Module 3 & 55 & D1100 x T224 \\
\hline Fold 4 Mirror & 0.8 & $256.8 \times 181.1 \times 25$ \\
\hline Double Fold Mirror & 8 & $200 \times 270 \times 38$ \\
\hline Collimator & 6.1 & $165 \times 200 \times 35$ \\
\hline Grating Turret & 79.9 & $386.375 \times 478.225 \times 340.675$ \\
\hline
\end{tabular}

\begin{tabular}{|l|c|c|}
\hline Module 4 & 54 & D1100 x T224 \\
\hline Cross Disperse turret & 50 & D209 x T198.425 \\
\hline
\end{tabular}

\begin{tabular}{|l|c|c|}
\hline Module 5 & 58.1 & D1100 x T224 \\
\hline Fold 7 Mirror & 5.4 & $289.125 \times 216.4 \times 42.175$ \\
\hline Interface Plate & 25.5 & D533.4 x T30.25 \\
\hline \multicolumn{2}{|c|}{} \\
\hline Camera Module & 73.8 & D400 x L393.75 \\
\hline Camera & 85 & \\
\hline
\end{tabular}




\subsection{Overall Support Structure}

The integrated support structure of GNIRS, as shown in Figure 9, comprises the G10 cylinder, and five modules. For clarity, a half cut away of the structure and the optical assemblies is illustrated in the figure. The entire structure is held as a cantilever and mounted on the ISS plate of the telescope structure. The overall mass is $755 \mathrm{~kg}$, which includes a camera assembly mass of $150 \mathrm{~kg}$ (65 kg of the camera module and $85 \mathrm{~kg}$ of camera optical components and mounting supports).

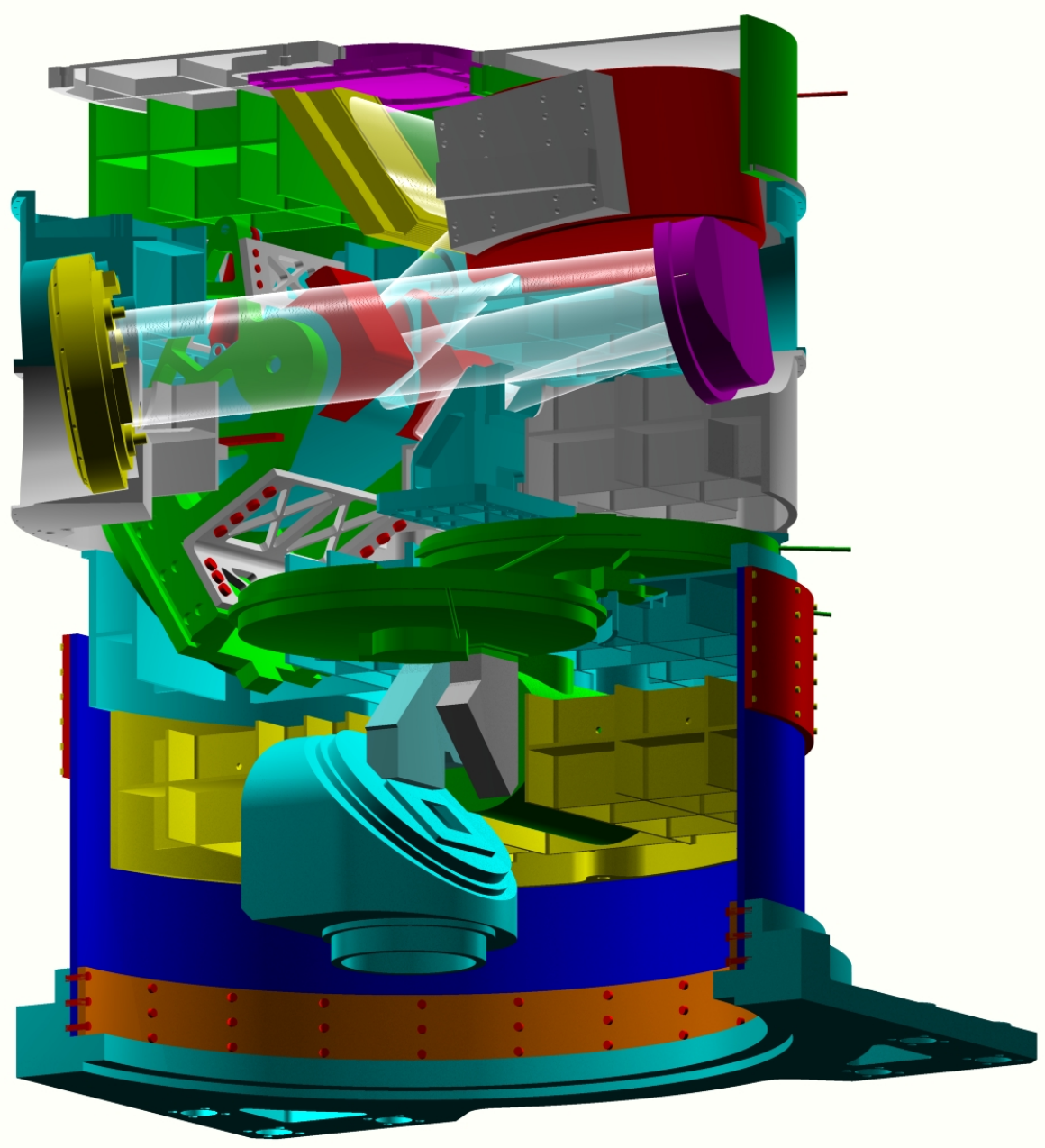

Figure 9. Integrated Support Structure of the GNIRS

A finite element model of the support structure was established. From frequency analysis, a fundamental frequency of $100 \mathrm{hz}$ was calculated. The first frequency is in a very traditional mode of a cantilever structure, as shown in Figure 10. This high frequency indicated that the support structure is adequately stiff.

Self-weight induced static structural analysis was conducted. This static analysis was used to determine the structural deflections of all the optical components for the gravity vector orientations in $\mathrm{X}, \mathrm{Y}$, and $\mathrm{Z}$, respectively. The global coordinate system used for GNIRS has the $\mathrm{X}$ direction aligned with the slit direction, the $\mathrm{Y}$ is the lateral direction, and the $\mathrm{Z}$ axis is parallel to the optical light path at the entrance window. For the lateral gravity cases (gravity along $\mathrm{X}$ or $\mathrm{Y}$ direction), a maximum deflection of 41 microns was obtained at the detector location. A relative deflection of 30 microns between the detector and the slit location was calculated. For the gravity along the optical axis ( $\mathrm{Z}$ axis), the effect is considerably smaller. The deformed information for these three orthogonal directions is implemented in the image motion evaluation. The relative motions of each individual optical component are ray traced for the calculation of the image motion. 
Maximum stress over the structure in any gravity orientations is $7 \mathrm{Mpa}(1,000 \mathrm{psi})$. The level of stress in the structure is considerably low comparing to the yield stress and the micro yield stress of AL T6061-T651 ${ }^{3}$. Consequently, there is not any significant stress concentration over the modules.

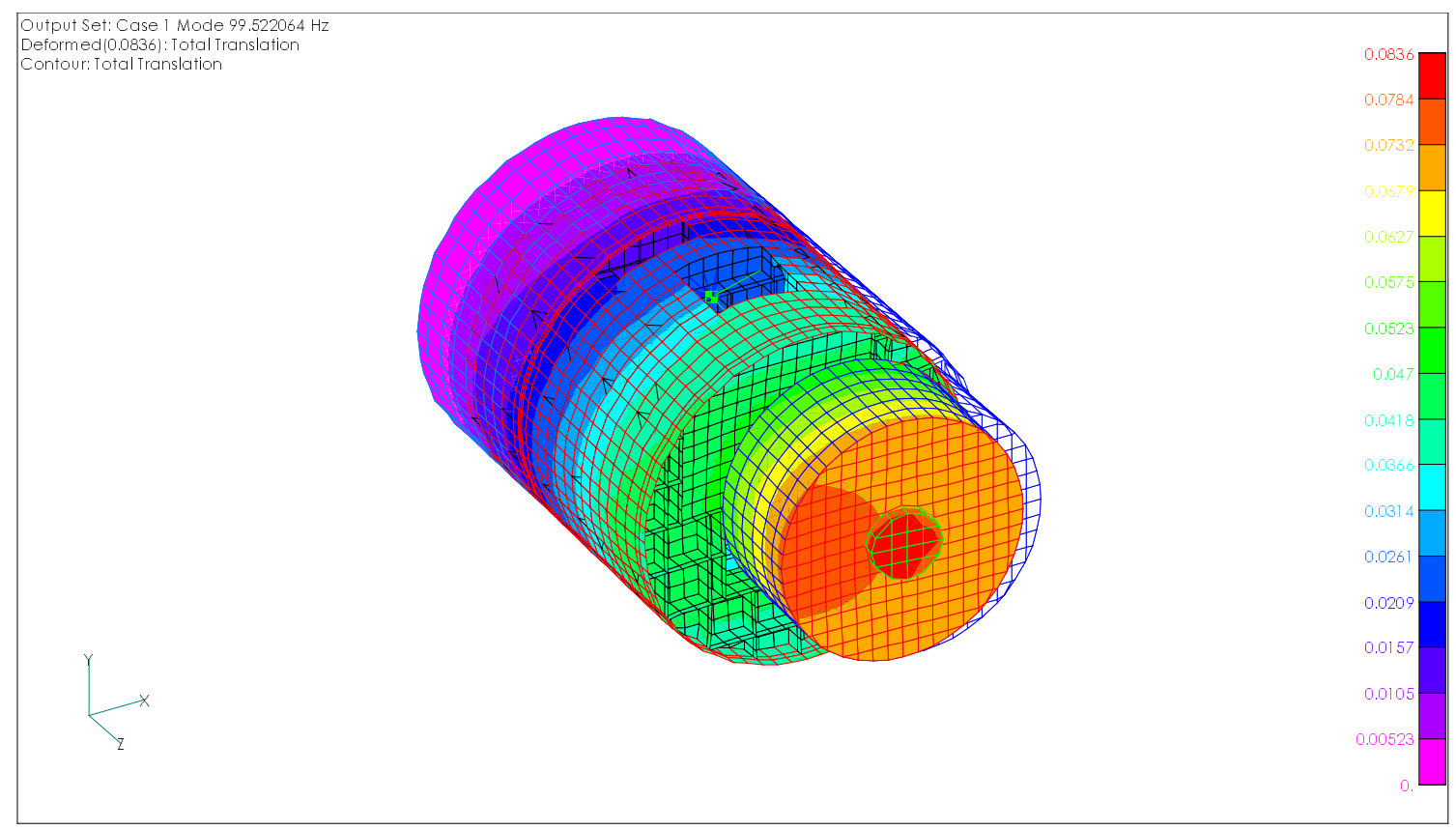

Figure 10. Fundamental Frequency and Mode Shape

It is noted that all of the analysis results are expressed in the global coordinate system. For a precise description of the changes in orientation of the optical components, it is necessary to define the optical displacements in each component's own coordinate system (local system). Hence, a proper coordinate transformation was required for each optical component. A typical structural deflection due to gravity in $\mathrm{Y}$ is listed in Table 3. Each optical component has 6 degrees of freedom, deflections are in $\mathrm{mm}$, and rotations are in mrad. The deflections of all optical components in the local system are evaluated and then the relative deflections between the successive components are obtained. The reason for computing the differences between successive components is that only the relative motion between successive components is relevant in this investigation.

\section{IMAGE MOTION ANALYSIS}

In the design specification, the image motion variation was limited to 0.1 pixel, or about 2.7 microns as a maximum permissible slit image motion at the detector during a one hour exposure. The goal is to keep the image stable within $1 / 10$ of a pixel size of $27 \mathrm{~mm}$, or $2.7 \mathrm{~mm}$ during a one-hour observation. Image movement on the slit should also be kept to less than $3 \mathrm{~mm}$ in a direction normal to the slit in order to maintain less than $5 \%$ vignetting during an one hour observation. (Noted that the motion along the slit causes no vignetting). This specification on the flexure of the instrument corresponds to the effects of a 15 degree rotation of the gravity vector during the period of observation.

The motion of each optical element due to self-weight deflection contributes to a shift of the image on the detector. A detailed optical analysis on the effect of each single component's motion in relation to the image motion on the detector has been carried out ${ }^{6}$. This image motion calculation is a first order approximation that combines the data from the structural deflection due to the gravity with the optical sensitivity analysis. This provides a global indication of the performance and an estimate of the magnitude of image motion both on the detector and on the slit as the gravity vector changes. 
Lateral gravitational deflections appeared most dominate, as seen in Table 3. The corresponding gravity orientation was considered for the image motion calculation. For the telescope orientation, a first order image motion can be defined as:

Table 3. A typical deflections due to gravity in $Y$ axis

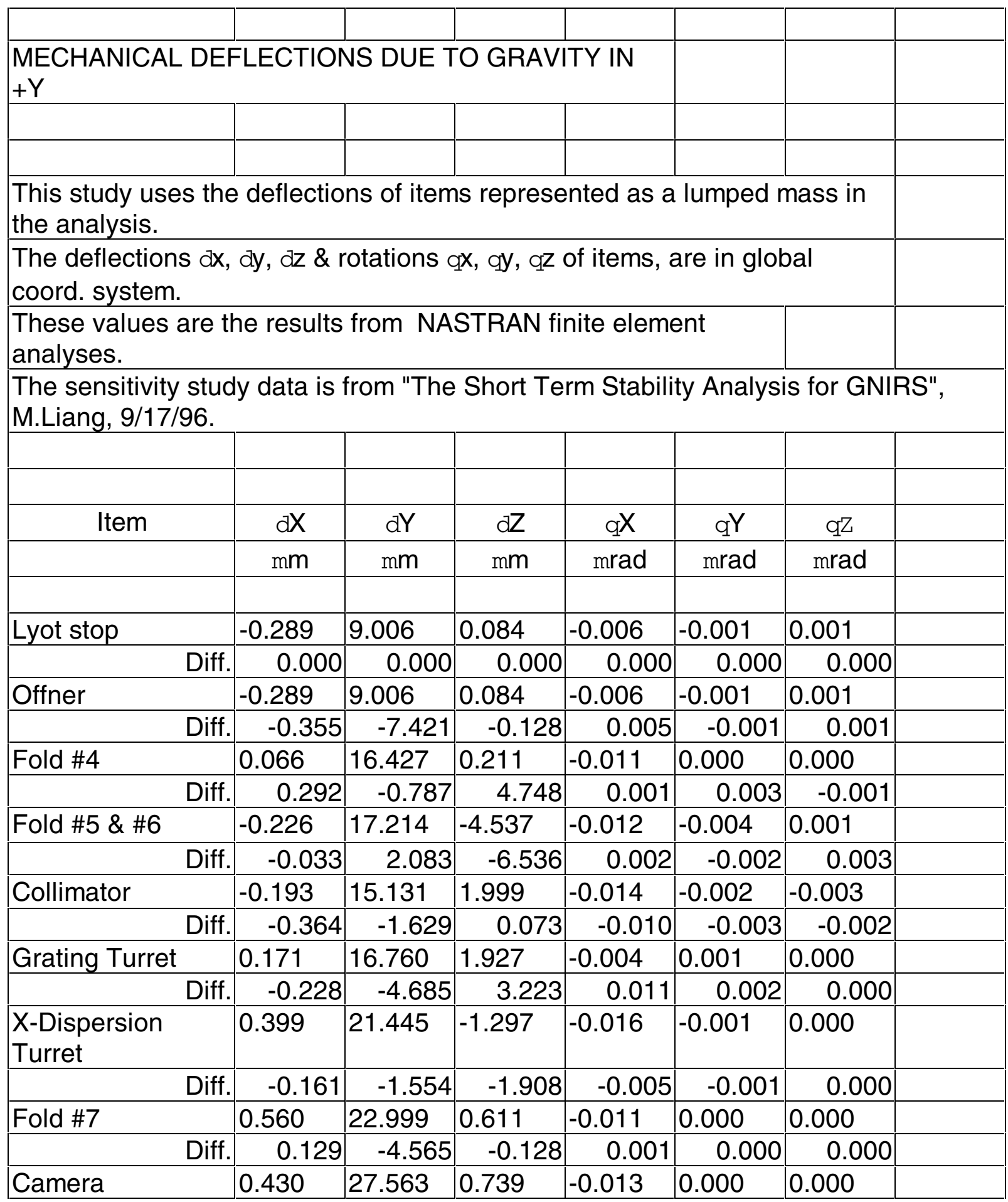




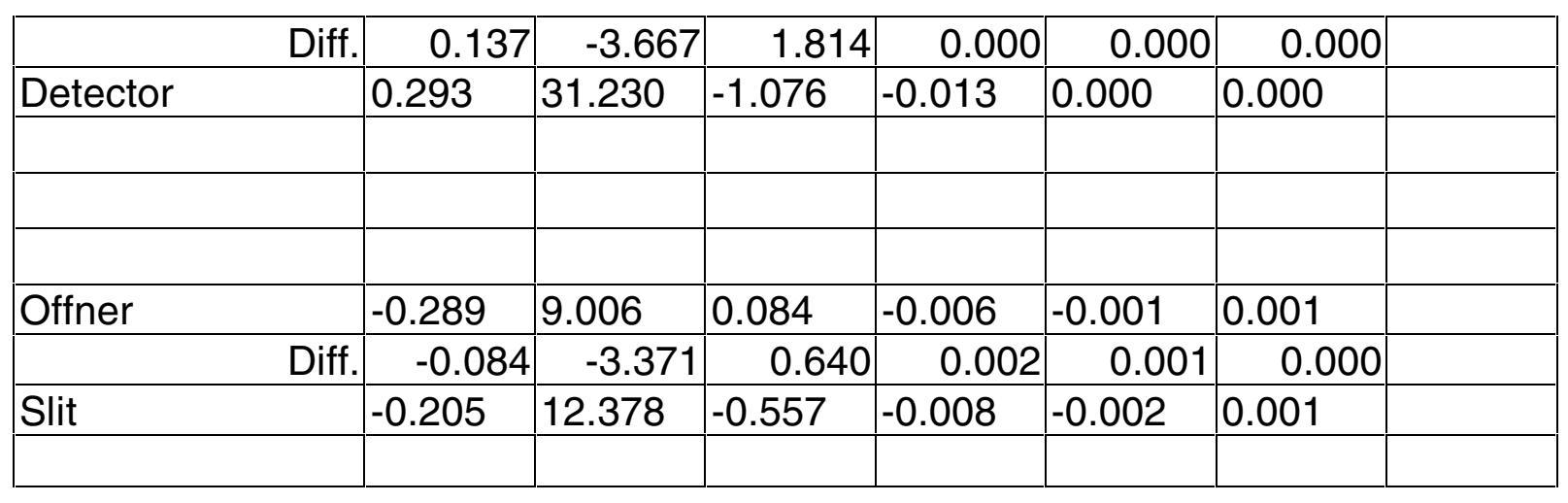

$$
\text { Image motion }=\operatorname{sqrt}\left[h_{x^{2}}^{2 *}(\cos f-\cos q)^{2}+h_{y}^{2 *}(\sin f-\sin q)^{2}\right]
$$

,where

$\mathrm{hx}=$ combined image motion with gravity in $\mathrm{X}$ direction

hy $=$ combined image motion with gravity in $\mathrm{Y}$ direction

$\mathrm{f}=$ angular position where calibration takes place, in this case, use $\mathrm{f}=0^{\circ}$ or at zenith pointing

$q=$ telescope position where image motion takes place, use $q=15^{\circ}$

The image motion on the detector is shown in Figure 11. The total image motion from zenith to horizon is $5.0 \mathrm{~mm}$. From any 15-degree range, the image motion is about $1 \mathrm{~mm}$, less then $1 / 10$ of the pixel size of $2.7 \mathrm{~mm}$. In the 90 -degree range, the total motion is estimated to be about $5 \mathrm{~mm}$.

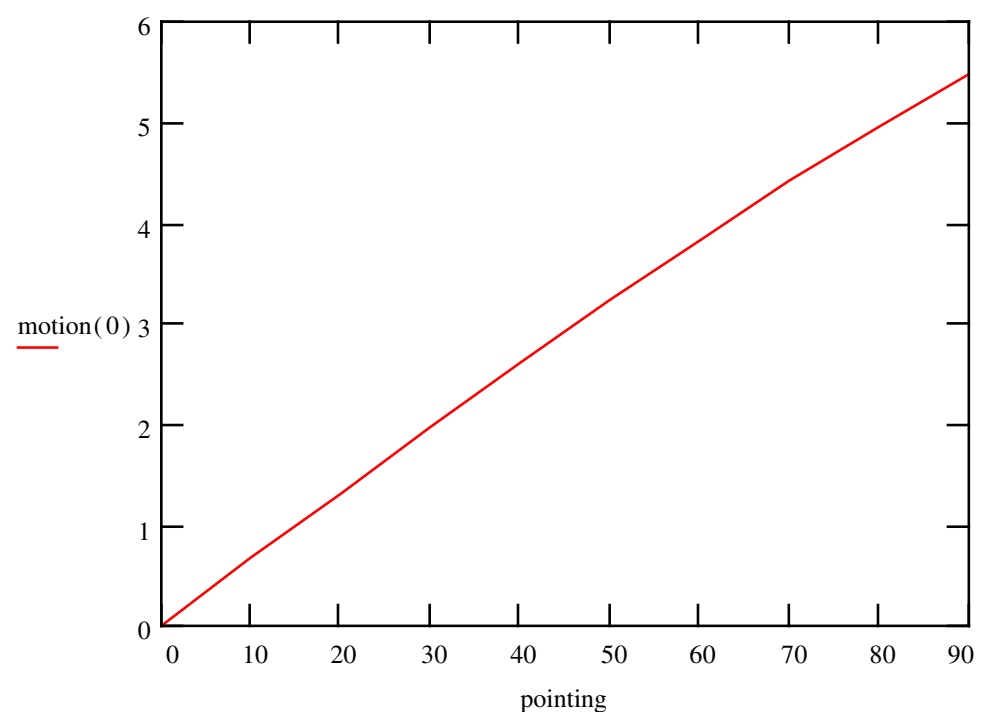

Figure 11. Image motion on detector (in $\mathrm{mm}$ ) vs. change of gravity vector (in degree).

The image motion on the slit is calculated in the same fashion as the image motion on the detector. Note that the deflection along the slit causes no vignetting. Again assuming the instrument is calibrated 
with the telescope in zenith position, the image motion at the slit location is shown in Figure 12 . The total image motion from zenith to horizon in this case is $11.5 \mathrm{~mm}$. Or, with the most conservative scenario and over a 90-degree range, the vignetting is only slightly above $10 \%$. In any 15-degree range, this motion is estimated to be about $3 \mathrm{~mm}$. A $3 \mathrm{~mm}$ motion is equivalent to a $5 \%$ vignetting. In a 90degree range, the motion is estimated to be $11 \mathrm{~mm}$. Note that this deflection is relative to the mounting plate and does not therefore include the effects of compensation by the OIWFS. Hence, It is a worst case analysis.

\section{SUMMARY AND CONCLUSIONS}

A parametric design study was conducted to determine an optimum configuration of the support structure for the GNIRS. In order to achieve athermalization of the instrument, the same material for the entire instrument was employed. All the reflective optics, the mounting mechanism, and the support structure of the GNIRS were designed with aluminum alloy AL6061-T6. For a minimum stress induced from mounting the optics, the optical components were installed with the help of semikinematic mirror mounts and flexure mechanisms.

The overall mass of the GNIRS support structure including the optics was estimated to be $755 \mathrm{~kg}$. The fundamental frequency is $100 \mathrm{hz}$. These results indicated that the support structure is capable of carrying the instruments and is stable. For a first order approximation in the image motion evaluation, a 15-degree motion of the telescope along the meridian causes the image to move $0.991 \mathrm{~mm}$ on the detector. In a 90-degree change of gravity vector, the motion is about 0.2 pixel size, or $5.0 \mathrm{~mm}$. Also, the structural stiffness is sufficiently high that the estimated image movement across the slit is $3 \mathrm{~mm}$ over a 15-degree change of gravity vector. This is equivalent to a 5\% vignetting (Reference 6).

Over an entire 90-degree change in gravity vector, the relative motion between the image and the slit is estimated to be $11.5 \mathrm{~mm}$. Due to the intrinsic stiffness of the optical component support structure, the gravity effects on the instrument would not produce any significant affect on the optical performance. The image motions due to self-weight deflection were within the error budget. It was found that large error contributions to the image were caused by the Offner, the collimator, and the camera assembly.

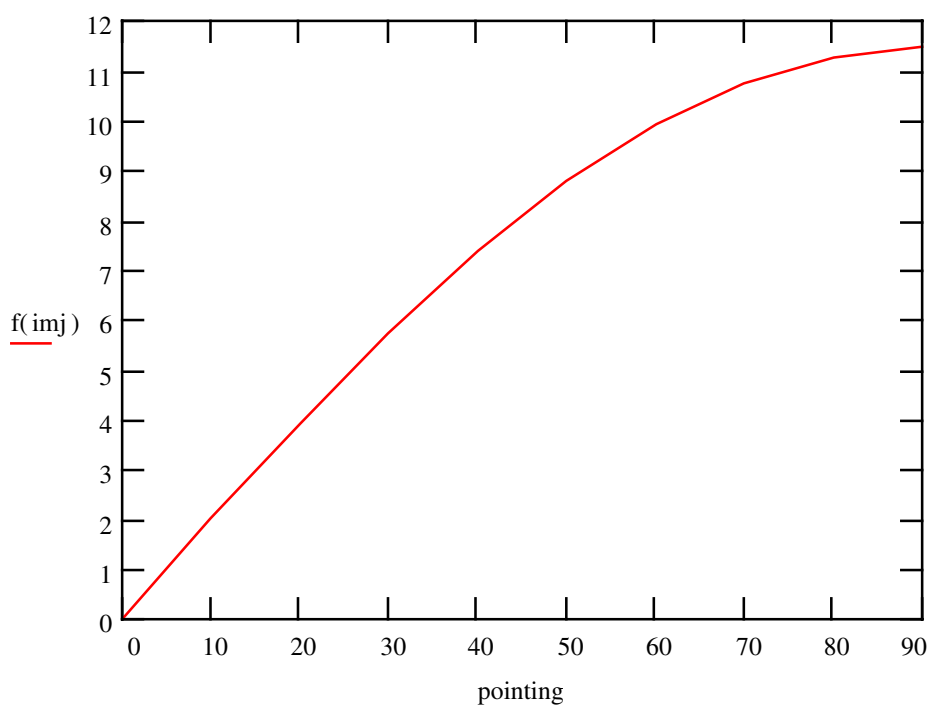


Figure 12. Image motion normal to the slit in $\mathrm{mm}$ vs. change of gravity vector in degree.

\section{ACKNOWLEDGEMENT}

The research in this paper was funded by the International Gemini 8-m Telescopes Project. The Gemini 8-m Telescopes Project is managed by the Association of Universities for Research in Astronomy for the National Science Foundation, under an international partnership agreement.

The authors would like to thank Daniel Vukobratovich and Jay Elias for their helpful comments and discussions.

\section{REFERENCES}

1. J. B. Hearnshaw, "The analysis of starlight: one hundred and fifty years of astronomical spectroscopy", Cambridge Press, New York, 1986.

2. M. Serrurier, "Structural features of the 200-inch telescope for Mt. Palomar Observatory", Civil Engineering, Vol. 8, No. 8, S24, August, 1938.

3. J. H. Faupel and F. E. Fisher, "Engineering Design", John Wiley \& Sons, New York, 1981.

4. W. C. Young, "Roark's Formulas for stress and strain”, 6 ed., McGraw-Hill Book Company, 1989.

5. Gemini Near Infrared Spectrograph Critical Design Review materials, NOAO, November 1997.

6. M. Liang, "The Short Term Stability Analysis for GNIRS", NOAO, September 1996.

7. Mark H. Lawry, "I-DEAS Master Series", Structural Dynamics Research Corporation, Millford, OH., 1997.

8. C. W. Marschall, M. E. Hoskins, and R. E. Maringer, "Stability of structural materials for spacecraft applications for the orbiting astronomical observatory project", NASA Goddard Space Flight Center, October 1969 